\title{
Perfil hematológico de codornas japonesas (Coturnix japonica) sob estresse térmico
}

\author{
Blood profile of japanese quail (Coturnix japonica) under thermal stress
}

\section{Gabriela do Amaral da Rosa ${ }^{\mathrm{I}}$ Luiz André Sorbello ${ }^{\mathrm{III}}$ Rosangela Locatelli Dittrich ${ }^{\mathrm{III}}$ Marcelo Tadeu Thomaz de Moraes ${ }^{\mathrm{II}}$ Edson Gonçalves de Oliveira ${ }^{\mathrm{II}}$}

RESUMO

$\mathrm{Na}$ coturnicultura, os limites das variáveis climáticas, como temperatura e umidade relativa do ar, interferem no desempenho produtivo e bem estar das aves. A temperatura de conforto térmico para codornas está entre 18 e $22^{\circ} \mathrm{C}$, sendo que o desconforto térmico pode causar estresse, alterando heterófilos e linfócitos circulantes e a relação heterófilo/linfócito. $O$ objetivo deste trabalho foi estabelecer os níveis de estresse em codornas durante os ciclos de produção com diferentes temperaturas, por meio das alterações no leucograma e da relação heterófilo-linfócito. O presente estudo foi realizado durante quatro ciclos de produção de 200 codornas japonesas (Coturnix japonica) com duração de 22 dias cada (denominados de C1, C2, C3 e C4). As temperaturas médias foram de: $22,4^{\circ} \mathrm{C}(\mathrm{C} 1) ; 25,5^{\circ} \mathrm{C}(\mathrm{C} 2) ; 23,3^{\circ} \mathrm{C}(\mathrm{C} 3) e$ $21,5^{\circ} \mathrm{C}(\mathrm{C} 4)$. Foram coletadas amostras de sangue de 80 codornas em cada um dos ciclos para determinação dos parâmetros hematológicos e dos valores da relação heterófilolinfócito $(H / L)$. As médias das relações heterófilo/linfócito foram 1,695 em C1; $2,424 \mathrm{em} \mathrm{C2;} \mathrm{1,8} \mathrm{em} \mathrm{C3} \mathrm{e} \mathrm{1,29} \mathrm{em} \mathrm{C4.}$ Comparando-se as médias das relações heterófilo/linfócito entre os quatro ciclos, tem-se que o valor de p foi inferior a 0,0001 , exceto entre a primeira e a terceira coleta, cujo valor de $p$ foi não significativo. A heterofilia e linfopenia verificadas $e$ o aumento da relação heterófilo/linfócito nos ciclos, coincidindo com o aumento da temperatura, além do limite de conforto térmico para estas aves, principalmente em C2, é uma evidência de que esses animais foram submetidos a estresse térmico e que as codornas são aves extremamente suscetiveis a variações climáticas.

Palavras-chave: coturnicultura, hematologia, temperatura.

\begin{abstract}
In quail raising the limits of climatic variables such as temperature and relative humidity, affect the performance and welfare of these birds. The thermal comfort temperature for quails is between 18 and $22^{\circ} \mathrm{C}$ and the discomfort can cause stress by changing circulating heterophils and lymphocytes and heterophil/ lymphocyte ratio. The objective of this study was to establish the stress levels in quails during the production cycles with different temperatures through changes in WBC and the heterophil/ lymphocyte ratio. This study was conducted in four production cycles of 200 Japanese quails (Coturnix japonica), during 22 days each one (referred as $\mathrm{C1}, \mathrm{C} 2, \mathrm{C} 3$ and $\mathrm{C} 4$ ). Average temperatures were: $22.4^{\circ} \mathrm{C}(\mathrm{C} 1), 25.5^{\circ} \mathrm{C}(\mathrm{C} 2), 23.3^{\circ} \mathrm{C}(\mathrm{C} 3)$ and $21.5^{\circ} \mathrm{C}(\mathrm{C} 4)$. We collected blood samples from 80 quails in each cycle for determination of haematological parameters and values of the heterophil-lymphocyte ratio $(H / L)$. The heterophil / lymphocyte ratio average was 1.695 for C1, 2.424 for C2, 1.8 for $C 3$ and 1.29 for C4. Comparing this average among the four cycles is noted that the $p$ value was less than 0.0001 , except between the first and third periods, in which the $p$ value was not significant. The heterophilia and lymphopenia verified and the increase in the heterophil/ lymphocyte ratio in the cycle, coinciding with rising temperatures, beyond the limit of thermal comfort for these birds mainly in $\mathrm{C2}$, is evidence that these birds were in heat stress and that the quails are highly susceptible birds to climate variations.
\end{abstract}

Key words: quail raising, hematology, temperature.

'Laboratório de Patologia Clínica Veterinária, Hospital Veterinário, Universidade Federal do Paraná (UFPR), 80035-050, Curitiba, PR, Brasil. E-mail: gabi.amaral.rosa @gmail.com. Autor para correspondência.

"Departamento de Zootecnia, UFPR, Curitiba, PR, Brasil.

II'Departamento de Medicina Veterinária, UFPR, Curitiba, PR, Brasil. 


\section{INTRODUÇÃO}

A situação atual da coturnicultura brasileira revela o início de uma nova fase no país, com superação do período de amadorismo, para solidificação como exploração industrial (LEANDRO, 2005). Sua expansão ocorre em função dos fatores como o potencial das codornas para a produção de ovos, pequena exigência de espaço, baixo consumo de ração, curto ciclo de produção, maturidade sexual precoce, grande resistência a enfermidades e baixo investimento inicial à produção com rápido retorno do capital (TDNET, 2006). De acordo com o IBGE (2002), a região sudeste detém aproximadamente $67 \%$ da produção nacional, destacando-se o estado de São Paulo com quase metade da coturnicultura brasileira.

As variáveis ambientais, como temperatura e umidade relativa do ar (UR), interferem no desempenho produtivo e bem estar das aves. Em condições de bemestar pobre, as aves respondem com alterações fisiológicas caracterizadas por elevada taxa cardíaca, aumento na liberação de corticosterona e de catecolaminas, anormalidades da adrenal e imunossupressão (NORDI et al., 2007). De acordo com MAXWELL (1993) e BORGES et al. (2003), o sistema sanguíneo é particularmente sensível às mudanças de temperatura e se constitui em importante indicador das respostas fisiológicas da ave a agentes estressores, havendo alterações quantitativas e morfológicas nas células sanguíneas. O estresse provocado por desconforto térmico induz o aumento dos níveis de corticosterona circulante, suprime a imunidade humoral e altera os valores de leucócitos circulantes em aves, com concomitantes alterações na resistência a doenças e decréscimo no crescimento e produção de ovos (ONBASILAR, 2005). Apesar dos prejuízos que podem ser causados pelo estresse em aves de produção, como frangos e perus, os estudos a respeito da mensuração de seus níveis em codornas são escassos.

Em climas tropicais e subtropicais como os do Brasil, a radiação solar intensa e a elevada temperatura e umidade relativa do ar no verão geram desconforto térmico quase permanente às aves, prejudicando seu desempenho produtivo e tornandose um dos principais problemas que afetam a exploração avícola (TINOCO, 1994). Altas temperaturas afetam o desempenho dos animais, reduzindo o consumo de ração e de nutrientes essenciais às funções fisiológicas (TINOCO, 1994; BORGES et al., 1997, 2001, 2003; JUNQUEIRA et al., 2000).

Na grande maioria das aves, heterófilos são as segundas células mais numerosas na circulação, havendo um aumento durante condições de estresse leve a moderada. A relação heterófilo:linfócito (H:L) é um parâmetro eficiente para a mensuração de grau de bem-estar em galinhas (GROSS \& SIEGEL, 1983). A relação heterófilo/linfócito é alterada como consequência do aumento de heterófilo e redução de linfócito, e tem sido proposta como um índice sensível ao estresse em aves (MAXWELL, 1993; BORGES, 1997, 2001).

As codornas são aves extremamente exigentes quanto aos limites das variáveis climáticas. $\mathrm{Na}$ fase de postura, a faixa ideal de temperatura situase entre 18 a $22^{\circ} \mathrm{C}$ (MAS et al., 2004). Segundo SINGH \& NARAYAM (2002), o intervalo de temperatura ideal para codornas a partir da quarta semana de idade é de 21 a $25^{\circ} \mathrm{C}$ e a umidade relativa do ar desejada para esta criação situa-se em torno de $60 \%$.

Com relação à densidade de aves alojadas em gaiolas, segundo PAVAN et al. (2005), no Brasil, é prática comum aumentar o número de aves por gaiola, devido à demanda e economia no processo de produção. Poucos estudos têm sido realizados sobre a relação entre a densidade das gaiolas utilizadas e o desempenho das aves durante a produção, mas sabese que a alta densidade é um fator que pode causar estresse às aves, sendo um fator de grande importância econômica. Recomenda-se, para a criação de codornas japonesas, na fase de postura, uma área de $100 \mathrm{~cm}^{2} \mathrm{ave}^{-1}$ (MURAKAMI \& ARIKI, 1998; LEANDRO et al., 2005).

$\mathrm{O}$ fator estresse e suas consequências na criação de frangos e perus tem sido avaliado, porém o monitoramento e o diagnóstico do estresse em codornas são escassos e, até o presente, inexistem estudos correlacionando estresse com temperatura e umidade relativa do ar. Dessa forma, os objetivos do presente estudo foram monitorar os parâmetros hematológicos e de proteína plasmática total de codornas submetidas a diferentes ambientes, estabelecer as proporções de heterófilo:linfócito para contribuir no conhecimento dos fatores de estresse em aves e melhorar a criação e manejo de codornas.

\section{MATERIAL E MÉTODOS}

O experimento foi realizado no Setor de Animais Alternativos e Silvestres no Centro de Estações Experimentais da UFPR, no período de dezembro de 2008 a abril de 2009.

Foram utilizadas 200 codornas (Coturnix japonica), adquiridas com cinco semanas de idade, que passaram por período de adaptação de três semanas, tendo, portanto, oito semanas de idade no início do experimento. 
As codornas foram alojadas em galpão semiaberto de alvenaria, com mureta lateral de $120 \mathrm{~cm}$ de altura, tela até o teto e cobertura de telhas de cimento amianto. Foram dispostas em gaiolas de arame galvanizado medindo $100 \times 50 \times 20 \mathrm{~cm}$ (largura $\mathrm{x}$ comprimento $\mathrm{x}$ altura) e divididas em cinco células iguais, com três aves por célula.

A água era fornecida por meio de bebedouros tipo copo, com válvula automática para manutenção do nível. Os comedouros, individuais para cada célula, eram manuais tipo calha. A função dos bebedouros era verificada diariamente e a ração fornecida à vontade, distribuída de manhã e à tarde. $\mathrm{O}$ experimento foi dividido em quatro ciclos de 22 dias, sendo denominados ciclos no verão.

Durante todo o período, foram registrados, diariamente, os valores de temperatura e umidade relativa ambiental, mensuradas com a utilização de um higrômetro. Foram registradas as temperaturas mínimas e máximas em diárias e obtidos os valores da temperatura média e temperaturas mínima e máxima médias em cada período. As temperaturas mínimas e máximas foram obtidas em cada ciclo. Foram registrados os valores da umidade relativa (UR) do ar mínimos e máximos, para obter a UR média por período.

Foram realizadas quatro coletas de sangue, uma a cada ciclo. As amostras de sangue foram obtidas por punção da veia jugular, em seringas heparinizadas, e acondicionadas em tubos. As extensões foram realizadas imediatamente após a coleta e, posteriormente, coradas com corante de Wright. As análises hematológicas foram realizadas no Laboratório de Patologia Clínica Veterinária da UFPR. O hematócrito foi determinado pela técnica do microhematócrito. As contagens de eritrócitos e leucócitos foram realizadas em Câmara de Neubauer, com diluição prévia do sangue (1:100) em azul de cresil (THRALL, 2004). A relação heterófilo:linfócito foi obtida dividindo-se o número de heterófilos pelo número de linfócitos (SCHALM'S, 2000). A proteína plasmática foi determinada pelo método do refratômetro. Os resultados foram analisados por meio do teste ANOVA, com a utilização do programa StatView.

\section{RESULTADOS E DISCUSSÃO}

Os resultados referentes às mensurações de temperatura e umidade relativa do ar durante cada ciclo produtivo avaliado estão na tabela 1 .

As temperaturas médias durante os ciclos foram de: $22,4^{\circ} \mathrm{C}(\mathrm{C} 1) ; 25,5^{\circ} \mathrm{C}(\mathrm{C} 2) ; 23,3^{\circ} \mathrm{C}(\mathrm{C} 3) \mathrm{e} 21,5^{\circ} \mathrm{C}$ (C4). A umidade relativa do ar (UR) média em todos os períodos foi superior a $72 \%$, com média geral de $74,3 \%$.

Os valores hematológicos obtidos em cada ciclo estão demonstrados na tabela 2. Entre os ciclos, observam-se diferenças significativas $(\mathrm{P}<0,05)$ entre $\mathrm{o}$ primeiro e todos os demais períodos para os valores de eritrócitos, leucócitos totais e basófilos. Os valores relativos e absolutos de heterófilos, linfócitos, monócitos e relação heterófilo-linfócito foram significativamente distintos nos ciclos dois e quatro, com a maior e menor temperatura, respectivamente.

Os valores absolutos médios de heterófilos e linfócitos $\left(\mu \mathrm{L}^{-1}\right)$ observados em cada ciclo foram, respectivamente, de 5.399 e 3.485 (C1); 7.987 e 3.567 (C2); 7.448 e 4.616(C3); 6.176 e 5.115(C4). Em codornas japonesas, DITTRICH et al., (2000) verificaram valores para heterófilos de $5.872 \mu \mathrm{L}^{-1}$ e para linfócitos de 16.056 $\mu \mathrm{L}^{-1}$. No presente estudo, os valores de heterófilos em $\mathrm{C} 2, \mathrm{C} 3$ e $\mathrm{C} 4$ foram superiores aos de DITTRICH et al. (2000) e, em todos os ciclos, os valores de linfócitos foram inferiores, caracterizando heterofilia e linfopenia, as principais alterações do estresse crônico (FUDGE \& JOSEPH, 2000; DAVIS et al., 2008; LATIMER \&

Tabela 1 - Umidade relativa e temperaturas mínimas, máximas e médias nos ciclos de produção de codornas japonesas.

\begin{tabular}{|c|c|c|c|c|c|}
\hline & $1^{\mathrm{o}}$ ciclo & $2^{\circ}$ Ciclo & $3^{\circ}$ Ciclo & $4^{\circ}$ Ciclo & Média Ciclos \\
\hline UR* média $(\%)$ & 72,1 & 75,1 & 77,1 & 72,8 & 74,3 \\
\hline UR Mínima (\%) & 50,0 & 55,0 & 64,0 & 64,0 & - \\
\hline UR Máxima (\%) & 91,0 & 91,0 & 86,0 & 90,0 & - \\
\hline Temp ${ }^{* *}$ Média $\left({ }^{\circ} \mathrm{C}\right)$ & 22,4 & 25,5 & 23,3 & 21,5 & 23,1 \\
\hline Temp Mínima Média $\left({ }^{\circ} \mathrm{C}\right)$ & 19,1 & 20,4 & 18,0 & 15,2 & 18,2 \\
\hline Temp Mínima $\left({ }^{\circ} \mathrm{C}\right)$ & 16,0 & 17,0 & 12,0 & 11,0 & - \\
\hline Temp Máxima Média $\left({ }^{\circ} \mathrm{C}\right)$ & 25,7 & 30,5 & 28,5 & 27,7 & 28,1 \\
\hline Temp Máxima $\left({ }^{\circ} \mathrm{C}\right)$ & 31,0 & 35,0 & 31,0 & 35,0 & - \\
\hline
\end{tabular}

*UR = Umidade relativa do ar.

**Temp $=$ temperatura

$1^{\circ}$ ciclo - dezembro de 2008 a janeiro de $2009.2^{\circ}$ ciclo - janeiro a fevereiro de $2009.3^{\circ}$ ciclo - fevereiro a março de $2009.4^{\circ}$ ciclo - março a abril de 2009

Ciência Rural, v.41, n.9, set, 2011. 
Tabela 2 - Valores hematológicos e de proteína plasmática total (média \pm erro padrão) para codornas durante quatro ciclos de produção.

\begin{tabular}{|c|c|c|c|c|}
\hline \multirow{2}{*}{ Parâmetros sanguíneos } & \multicolumn{4}{|c|}{--- } \\
\hline & $\mathrm{C} 1(\mathrm{a})$ & $\mathrm{C} 2(\mathrm{~b})$ & $\mathrm{C} 3(\mathrm{c})$ & $\mathrm{C} 4(\mathrm{~d})$ \\
\hline Hematócrito (\%) & $39,5 \pm 0,46$ & $39,1 \pm 0,37^{\mathrm{d}}$ & $39,0 \pm 0,34^{\mathrm{d}}$ & $40,4 \pm 0,35^{\mathrm{b}, \mathrm{c}}$ \\
\hline Eritrócitos $\left(\times 10^{6} \mu \mathrm{L}^{-1}\right)$ & $2,6 \pm 0,049^{\mathrm{b}, \mathrm{c}, \mathrm{d}}$ & $2,9 \pm 0,054^{\mathrm{a}}$ & $2,9 \pm 0,045^{\mathrm{a}}$ & $3,0 \pm 0,05^{\mathrm{a}}$ \\
\hline Leucócitos totais $\left(\mu \mathrm{L}^{-1}\right)$ & $9.762,5 \pm 483,7^{\mathrm{b}, \mathrm{cd}}$ & $12.343,9 \pm 582,7^{\mathrm{a}}$ & $12.930,1 \pm 675,5^{\mathrm{a}}$ & $12.062,5 \pm 515,7^{\mathrm{a}}$ \\
\hline Heterófilos $(\%)$ & $55,3 \pm 1,1^{\mathrm{b}, \mathrm{d}}$ & $64,7 \pm 1,1^{\mathrm{a}, \mathrm{c}, \mathrm{d}}$ & $57,6 \pm 1,2^{\mathrm{b}, \mathrm{d}}$ & $51,2 \pm 0,9^{\mathrm{a}, \mathrm{b}, \mathrm{c}}$ \\
\hline Linfócitos (\%) & $35,7 \pm 0,9^{\mathrm{b}, \mathrm{d}}$ & $28,9 \pm 0,9^{\mathrm{a}, \mathrm{c}, \mathrm{d}}$ & $35,7 \pm 1,0^{\mathrm{b}, \mathrm{d}}$ & $42,4 \pm 0,9^{\mathrm{a}, \mathrm{b}, \mathrm{c}}$ \\
\hline Eosinófilos (\%) & $1,4 \pm 0,1$ & $1,3 \pm 0,1$ & $1,4 \pm 0,1$ & $1,7 \pm 0,2$ \\
\hline Monócitos (\%) & $3,5 \pm 0,3^{\mathrm{b}, \mathrm{d}}$ & $2,3 \pm 0,2^{\mathrm{a}, \mathrm{c}}$ & $2,9 \pm 0,3^{b}$ & $2,5 \pm 0,2^{\mathrm{a}}$ \\
\hline Basófilos (\%) & $4,0 \pm 0,3^{b, c, d}$ & $2,9 \pm 0,2^{\mathrm{a}, \mathrm{d}}$ & $2,3 \pm 2,1^{\text {a }}$ & $2,2 \pm 0,2^{\text {a.b }}$ \\
\hline Relação Heterófilo:Linfócito & $1,7 \pm 0,1^{\mathrm{b}, \mathrm{d}}$ & $2,4 \pm 0,1^{\mathrm{a}, \mathrm{c}, \mathrm{d}}$ & $1,8 \pm 0,9 a^{b, d}$ & $1,3 \pm 0,1^{\mathrm{a}, \mathrm{b}, \mathrm{c}}$ \\
\hline Proteínas Plasmáticas Totais $\left(\mathrm{g} \mathrm{dl}^{-1}\right)$ & $6,1 \pm 0,2^{\mathrm{d}}$ & $6,0 \pm 0,2^{\mathrm{d}}$ & $6,5 \pm 0,1$ & $6,7 \pm 0,2^{\mathrm{a}, \mathrm{b}}$ \\
\hline
\end{tabular}

Médias \pm erro padrão seguidas de $\mathrm{a}, \mathrm{b}, \mathrm{c}$ ou d representam diferença significativa $(\mathrm{P}<0,05)$ com o respectivo ciclo, pelo teste Anova, Statview, SAS

Temperaturas médias: $22,4^{\circ} \mathrm{C}(\mathrm{C} 1) ; 25,5^{\circ} \mathrm{C}(\mathrm{C} 2) ; 23,3^{\circ} \mathrm{C}(\mathrm{C} 3)$ e $21,5^{\circ} \mathrm{C}(\mathrm{C} 4)$.

BIENZLE, 2010). A heterofilia também ocorre na inflamação, porém, doze horas após o início da resposta inflamatória, os valores de heterófilos são quatro vezes superiores aos parâmetros de referência (LATIMER et al., 1988; LATIMER et al.1989; LATIMER \& BIENZLE, 2010). No presente estudo, não foram observados heterófilos imaturos (bastonetes) e nem heterófilos tóxicos, alterações que aparecem com frequência nas aves doentes (LATIMER et al., 1988; LATIMER et al., 1989; LIND et al.,1990). Dessa forma, pode-se descartar a inflamação devido ao estado hígido dos animais, sem sinais clínicos sugestivos de doença, e pela ausência das alterações hematológicas condinzentes com resposta inflamatória.

As médias das relações heterófilo/linfócito, parâmetro enfocado no presente estudo, foram de 1,7 em C1; 2,4 em C2; 1,8 em C3 e 1,3 em C4. Comparandose as médias das relações heterófilo/linfócito entre os quatro ciclos, tem-se o valor de p inferior a 0,0001 (variação significativa), sendo que, apenas entre a primeira e a terceira coleta, o valor de $\mathrm{p}$ foi superior a 0,3 (não significativo). Em frangos, a proporção H:L superior a 1:3 geralmente indica início de doença (GROSS \& SIEGEL, 1993), o que não foi observado nas codornas deste estudo.

Os valores de hematócrito foram diferentes na análise dos valores entre $\mathrm{C} 4$ com $\mathrm{C} 2 \mathrm{e} \mathrm{C} 3$, enquanto os números de eosinófilos foram semelhantes em todos os ciclos, sem diferença significativa.

Os parâmetros hematológicos de codornas japonesas foram citados por RITCHIE et al. (1994), com valores de hematócrito de 37-46 (\%); eritrócitos de 3,34,1 $\left(\times 10^{6} \mu \mathrm{l}^{-1}\right)$; heterófilos de 20,8-52 (\%) e linfócitos de 40-73,6(\%).
Em estudo realizado no Paraná, DITTRICH et al. (2000) encontraram valores hematológicos para codornas (Coturnix coturnix) com número de

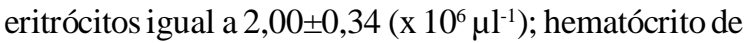
$39,64 \pm 4,3(\%)$ e proteínas plasmáticas de $3,83 \pm 1,06$ (g $\left.\mathrm{dl}^{-1}\right)$. Obteve-se, no mesmo estudo, número médio de leucócitos totais de 21.590, percentagem de heterófilos igual a $27,2 \%$ e de linfócitos igual a $74,37 \%$. Os valores de proteína plasmática total do presente estudo foram superiores (média de 6,0 $\mathrm{g} \mathrm{dl}^{-1}$ ) aos valores de DITTRICH et. al. (2000), mas as notáveis diferenças entre alguns parâmetros podem ser atribuídas a alguns fatores, como as espécies, dietas e níveis de estresse diferentes entre os dois estudos.

Os resultados obtidos neste trabalho foram valores relativos de linfócitos inferiores às referências propostas por RITCHIE et. al. (1994) e DITTRICH et. al. (2000). Da mesma maneira, os valores de heterófilos para todos os ciclos, em especial no segundo, encontraram-se no limite superior ou acima dos valores referenciados por RITCHIE et al. (1994) e DITTRICH et al. (2000). Essas variações podem ser devido aos níveis de estresse nos animais avaliados no presente estudo. O número de linfócitos diminui nas condições de estresse nas aves, devido à liberação de corticosterona endógena (HOLT, 1992; FUDGE \& JOSEPH, 2000; NORDI, 2007).

Segundo GROSS E SIEGEL (1993), há três valores que fundamentados na proporção heterófilo:linfócito podem ser utilizados para caracterizar os níveis de estresse em frangos de corte, indicando estresse. Nessa classificação, o valor 0,2 indicaria grau leve de estresse; 0,5 estresse 
intermediário ou moderado e 0,8 estresse alto. Em todos os ciclos de produção, extrapolando a classificação de GROSS \& SIEGEL (1994) para codornas, observa-se nível alto de estresse. O estresse severo não foi correlacionado à basofilia em cada ciclo, conforme descreveu MAXWELL (1993). É notável nos resultados que a relação heterófilo:linfócito aumenta com o aumento da temperatura média ambiental, coincidindo com o menor valor desta proporção em $\mathrm{C} 4\left(21,5^{\circ} \mathrm{C}\right)$ e a maior relação em $\mathrm{C} 2\left(25,5^{\circ} \mathrm{C}\right)$. Embora existam diferenças quanto à faixa de temperatura ideal à coturnicultura, de 18 a $22^{\circ} \mathrm{C}$ para MAS et al. (2004) e de 21 a $25^{\circ} \mathrm{C}$ para SINGH \& NARAYAM (2002), nota-se que em todos os ciclos a temperatura ultrapassou o nível de conforto térmico para a espécie avaliada. Em todos os ciclos, a umidade relativa do ar foi superior aos $60 \%$ propostos por SINGH \& NARAYAM (2002) para codornas adultas, revelando que o ambiente não era favorável ao bem-estar dessas aves.

A relação heterófilo:linfócito $(\mathrm{H} / \mathrm{L})$ é considerada o indicador mais sensível de estresse em aves do que os níveis plasmáticos de glicocorticóide (DAVIS et al, 2008), entretanto, a heteropenia e basofilia podem ocorrer durante o estresse severo, de acordo com MAXWELL (2003). Neste estudo, a heteropenia e basofilia não foram observadas.

O estresse observado nas codornas não está relacionado ao aumento da densidade populacional, porque o espaço existente no alojamento das aves foi mais que três vezes superior à área de $100 \mathrm{~cm}^{2}$ ave $^{-1}$ sugerida por MURAKAMI E ARIKI (1998) como ideal.

\section{CONCLUSÃO}

A heterofilia, a linfopenia e as diferenças observadas nas relações heterófilo:linfócito entre os ciclos permitem concluir que existe aumento dos níveis de estresse em codornas mantidas em condições de temperatura e umidade relativa do ar superiores às de conforto térmico. A inflamação não foi verificada devido à ausência de sinais clínicos e de alterações como desvio à esquerda (heterófilos imaturos), heterófilos tóxicos e heterofilia extrema.

\section{COMITÊ DE ÉTICA E BIOSSEGURANÇA}

O artigo foi aprovado pela Comissão de Ética no Uso de Animais da Instituição de origem, sob o protocolo 036/ 2008.

\section{REFERÊNCIAS}

BORGES, S.A. Suplementação de cloreto de potássio e bicarbonato de sódio para frangos de corte durante o verão. 1997. 84f. Dissertação (Mestrado em Zootecnia) -
Curso de Pós-graduação em Zootecnia, Universidade Estadual Paulista, SP.

BORGES, S.A. Balanço eletrolítico e sua interrelação com o equilíbrio ácido-base em frangos de corte submetidos a estresse calórico. 2001. 97f. Tese (Doutorado em Zootecnia) - Curso de Pós-graduação em Zootecnia, Universidade Estadual Paulista, SP.

BORGES, S.A. et al. Fisiologia do estresse calórico e a utilização de eletrólitos em frangos de corte. Ciência Rural, v.33, n.5, p.975-981, 2003. Disponível em: <http://www.scielo.br/ scielo.php? script $=$ sci_arttext \& pid = S $0103-$ 84782003000500028 >. Acesso em: 25 ago. 2010. doi: $10.1590 / \mathrm{S} 0103-84782003000500028$.

CAREY, J.B.; KUO, F.L. Effects of cage population on the productive performance of layers. Poultry Science, v.74, n.4, p.633-637, 1995. Disponível em: <http:// ww.scielo.br/ scielo.php?script=sci_arttext\&pid=S 1516 35982005000400029>. Acesso em: 25 ago. 2010. doi: $10.1590 / \mathrm{S} 1516-35982005000400029$.

DAVIS, A.K. et al. The use of leukocyte profiles to measure stress in vertebrates: a review for ecologists. Functional Ecology, v.22, p.760-772, 2008. Disponível em: <http:// www.mendeley.com/research/the-use-of-leukocyte-profiles-tomeasure-stress-in-vertebrates-a-review-for-ecologists/>. Acesso em: 03 ago.2009 doi: 10.1111/j.1365-2435.2008.01467.x.

DITTRICH, R.L. et al. Determinação do eritrograma e do proteinograma em codornas de Criação Industrial - Coturnix coturnix coturnix. In: CONBRAVET, 27.; CONPAVET, 5.; CONFERÊNCIA ANUAL DA SPMV, 60.; EXPOVET, 6., 2000, Águas de Lindóia - SP. Anais... São Paulo: SPMV, 2000. V.1, p. $10-11$

DITTRICH, R.L. et al. Leucograma AST e GGT em codornas de criação industrial - Coturnix coturnix coturnix - com alterações nos heterófilos. In: CONBRAVET, 27.; CONPAVET, 5.; CONFERÊNCIA ANUAL DA SPMV, VI EXPOVET, 55., 2000, Águas de Lindóia - SP. Anais... São Paulo: SPMV, 2000. V.1, p.10-10.

FLAUZINA, L.P. Desempenho produtivo e biometria de vísceras de codornas japonesas alimentadas com dietas contendo diferentes níveis de proteína bruta. 2007. $36 \mathrm{f}$. Dissertação (Mestrado em Ciências Agrárias) - Faculdade de Agronomia e Medicina Veterinária, Universidade de Brasília, DF.

GOUVEIA, F.C; DITTRICH, R.L. Determinação dos parâmetros hematológicos e bioquímicos em codornas de criação industrial - Coturnix coturnix coturnix. In: EVENTO DE INICIAÇÃO CIENTÍFICA - EVINCI, 2000, Curitiba. Anais... Curitiba: Universidade Federal do Paraná, Pró Reitoria de Pesquisa e Pós-Graduação, 2000. V.1, p.394-394.

GROSS, W.B.; SIEGEL, H.S. Evaluation of the heterophil/ lymphocyte ratio as a measure of stress in chickens. Avian Disease, v.27, n.4, p. 972-979, 1983.

GROSS, W.B.; SIEGEL, H.S. Effects of initial and second periods of fasting on heterophil/lymphocyte ratios and body weight. Avian Disease, v.30, p. 345-346, 1986. Disponível em: <http:/ / w w w. mendeley.com/research/evaluation- 
heterophillymphocyte-ratio-measure-stress-chickens-4/>. Acesso em: 03 ago. 2009. PMID: 6360120.

GROSS, W.B.; SIEGEL, H.S. General principles of stress and welfare. In: TEMPLE GRANDIN. Livestock handling and transport. Wallingford, UK.: CAB International, 1993. p.2134.

HOLT, P.S. Effects of induced moulting on immune response of hens. British Poultry Science, v.33. p.165-175, 1992. Disponível em: http://www.ncbi.nlm.nih.gov/pubmed/1571800. Acesso em: 03 ago. 2009. PMID: 1571800.

IBGE- Instituto Brasileiro de Geografia e estatística - Censo agropecuário 2002. Disponível em: 〈www.ibge.gov.br〉. Online. Acesso em: 15 jul. 2009.

JUNQUEIRA, O.M. et al. Efeitos das fontes e níveis de sódio, cloro e potássio e da relação $(\mathrm{Na}+\mathrm{K}) / \mathrm{Cl}$, sobre o desempenho e características do plasma sangüíneo de poedeiras comerciais. Revista Brasileira de Zootecnia, v.29, n.4, p.1110-1116, 2000. Disponível em: <http:/ $/$ www.scielo.br/scielo.php?script $=$ sci_arttext\&pid $=$ S151635982000000400023>. Acesso em: 03 ago. 2009. doi: 10.1590/ S1516-35982000000400023.

LATIMER K.S. et al. Ultrastructural changes in avian toxic heterophils. In: PROCEEDINGS ANNUAL MEETING OF ELECTRON MICROSCOPIC SOCIETY OF AMERICA, 46., San Francisco. Proceedings... San Francisco:L G. W. Bailey, ed.,1988. p.360-361.

LATIMER K.S., RAKICH P.M. Clinical interpretation of leukocyte responses. Veterinary Clinics North America: Small Animal Practice, v.19, p.637-668, 1989. Disponível em: <http://www.ncbi.nlm.nih.gov/pubmed/2672534>. Acesso em: 03 ago. 2009. PMID: 2672534.

LATIMER, K.S.; BIENZLE, D. Determination and Interpretation of the avian leukogram. In: FELDMAN, B.F. et al. SCHALM'S veterinary hematology. 5.ed. Philadelphia: Lippincott Williams \& Wilkins, 2000. Chapt.60, p.417-432.

LATIMER, K.S.; BIENZLE, D. Determination and interpretation of the avian leukogram. In: WEISS, D.J.E.; WARDROP K. SCHALM'S veterinary hematology. 6.ed.. Ames, IA: Blackwell Publishing, 2010. Chapt.50, p.345-357.

LEANDRO, N.S.M. et al. Desempenho produtivo de codornas japonesas (Coturnix coturnix japonica) submetidas a diferentes densidades e tipos de debicagem. Acta Scientiarum. Animal Sciences, v.27, n.1, p.129-135, 2005. Disponível em: <http:/ /periodicos.uem.br/ojs/index.php/ActaSciAnimSci/article/ viewFile/1263/690>. Acesso em: 05 ago. 2009. doi: 10.4025/ actascianimsci.v27i1.1263.

LIND, P.J. et al. Morphology of the eosinophil in raptors. Journal of the American Veterinary Medical Association, 1990: Chap. 4, p.33-38, 1990.

LINSLEY, J.G.; BURGER, R.E. Respiratory and cardiovascular response in the hyperthermic domestic cock. Poultry. Science, v.43, n.1, p.291-305, 1964.
MACARI, M. et al. Fisiologia aviária aplicada a frangos de corte. Jaboticabal: FUNEP/UNESP, 1994. 246p.

MAS, H.A.R. et al. Energia metabolizável de alimentos protéicos para codornas japonesas (Coturnix coturnix japonica). In: SIMPÓSIO INTERNACIONAL, 2.; CONGRESSO BRASILEIRO DE COTURNICULTURA, 2004, Lavras, MG. Anais... Lavras: Universidade Federal de Lavras, 2004. p.204

MAXWELL, M.H. Avian blood leucocyte responses to stress. British Poultry Science, v.49, p.34-43, 1993.

MURAKAMI, A.E.; ARIKI, J. Produção de codornas japonesas. Jaboticabal: Fundação de Apoio a Pesquisa, Ensino e Extensão, 1998. 79p.

NORDI, W.M. et al. Diagnóstico de bem-estar de codornas japonesas utilizando a liberdade sanitária em dois sistemas de criação. Curitiba: Labea Publicações, UFPR, 2007. 6p.

ONBASILAR, E.E.; AKSOY, F.T. Stress parameters and immune response of layers under different cage floor and density conditions. Livestock Production Science, Elsevier, v.95, p.255-263, 2005. Disponível em: <www.sagbilens.ankara.edu.tr/ items/Image/yayin/science.pdf>. Acesso em: 03 set. 2009. doi:10.1016/j.livprodsci.2005.01.006.

PAVAN, A.C. et al. Efeito da densidade na gaiola sobre o desempenho de poedeiras comerciais nas fases de cria, recria e produção. Revista Brasileira de Zootecnia, v.34, n.4, p.1320-1328, 2005. Disponível em: <http://www.scielo.br/ scielo.php? script=sci_art text\&pid=S 1516 35982005000400029>. Acesso em: 03 set. 2009. doi: 10.1590/ S1516-35982005000400029.

RITCHIE, B.W. et al. Avian medicine: principles and application. Lake Worth, Florida: Wingers, 1994. 1407p.

SINGH, R.V.; NARAYAN, R. Produção de codornas nos trópicos. In: SIMPÓSIO INTERNACIONAL DE COTURNICULTURA, 2002, Lavras, MG. Anais... Lavras: Universidade Federal de Lavras, 2002. p.27-36.

STRUWE, F.J. et al. Effect of rearing floor type and tenday beak trimming on stress and performance of caged layers. Poultry Science, v.71, p.70-75, 1992. Disponível em: <http:/ /www.ncbi.nlm.nih.gov/pubmed/1539024>. Acesso em: 03 set. 2009. PMID: 1539024.

TDNET - Aspectos na criação e manejo de codornas para postura. 2006. Disponível em: <http://www.tdnet.com.br/ TDS/TD/SCRIPT/DESCRICAO>. Acesso em: 29 jul. 2009.

THRALL, M.A. Veterinary hematology and clinical chemistry. Baltimore: Lippicott Williams \& Wilkins, 2004. $518 \mathrm{p}$.

TINOCO, I.F.F. Resfriamento adiabático evaporativo no controle térmico de galpões avícolas. In: CONFERÊNCIA APINCO DE CIÊNCIA E TECNOLOGIA, 1994, Campinas, SP. Anais... Campinas: Facta, 1994. p.129-133. 